5. World Health Organization. WHO Guidelines on tularaemia. Geneva, Switzerland: World Health Organization; 2007.

6. Splettstoesser WD, Tomaso H, Al Dahouk S, Neubauer H, SchuffWerner P. Diagnostic procedures in tularaemia with special focus on molecular and immunological techniques. J Vet Med B Infect Dis Vet Public Health 2005;52:249-261.

7. Jones RM, Nicas M, Hubbard A, Sylvester MD, Reingold A. The infectious dose of Francisella tularensis (tularemia). Appl Biosaf 2005; 10:227-239.

8. Tang JW, Li Y, Eames I, Chan PK, Ridgway GL. Factors involved in the aerosol transmission of infection and control of ventilation in healthcare premises. J Hosp Infect 2006;64:100-114.

9. Mesner O, Riesenberg K, Biliar N, et al. The many faces of humanto-human transmission of brucellosis: congenital infection and outbreak of nosocomial disease related to an unrecognized clinical case. Clin Infect Dis 2007;45:e135-e140.

\section{Port-Related Nontyphoidal Salmonella Bacteremia}

To the Editor-Salmonella species, a genus of the family Enterobacteriaceae, includes Salmonella enterica serovar Typhi and nontyphoidal Salmonella (NTS) spp. ${ }^{1,2}$ Human infections caused by NTS are often associated with contaminated food products and always develop in an immunocompromised host. $^{3}$ The clinical presentations of NTS infection include gastroenteritis, primary bacteremia, mycotic aneurysm, infective endocarditis, urinary tract infection, meningitis, empyema thoracis, and osteomyelitis. ${ }^{3-7}$ We performed a study to identify cases of unusual presentation of NTS infection related to subcutaneously implanted port reservoir and to further investigate their associated clinical and microbiological characteristics.

This study was conducted at 1 institution, a 900-bed hospital in southern Taiwan. From the computerized database of the bacteriology laboratory, we identified patients whose cultures yielded NTS. The medical records of all patients with portrelated infection caused by NTS included in this study were retrospectively reviewed.

The diagnosis of port-related NTS bacteremia was defined as a primary laboratory-confirmed NTS bacteremia in a patient with a port at the time of (or within 48 hours prior to) the onset of symptoms in whom the infection was not related to another site. Standard definitions for healthcare-associated bacteremia (HAIs) were used. ${ }^{8}$ Inappropriate use of antibiotics was defined as use of antimicrobial agents to which the clinical isolates were resistant in vitro.

During the study period, 4 patients were identified to have port-related NTS bacteremia: 3 infections were caused by group D Salmonella, and 1 was caused by group C Salmonella. All of the clinical isolates were susceptible to ampicillin, ceftazidime, ceftriaxone, ciprofloxacin, trimethoprim-sulfamethoxazole, and chloramphenicol. The clinical characteristics of 4 patients with port-related NTS bacteremia are summarized in Table 1. Of these 4 patients, 3 were men, and the age range of this cohort was $44-80$ years. All of these patients had various cancers, and 1 patient had received chemotherapy prior to NTS infection. All of these patients had initial presentations of fever; however, none had signs or symptoms of enteritis. In addition, 1 patient had diabetes mellitus. Of these 4 patients, 3 had white blood cell counts $>11,000 / \mathrm{mm}^{3}$, and none had neutropenia. In addition, 3 patients had hemoglobin $<10 \mathrm{~g} / \mathrm{dl}$, and 2 patients had elevated C-reactive protein levels. None of these patients had their port removed. Although all 4 patients received appropriate antibiotics initially, 1 patient died due to NTS sepsis.

This study describes a rare cluster of NTS bacteremia in hospitalized cancer patients with ports at a single center. The immunocompromised conditions among these patients should be included as major risk factors for NTS bacteremia. Moreover, all of cases in this survey were classified as healthcare-associated, catheter-related bloodstream infections. Although rare, NTS should be considered as a possible pathogen causing intravascular catheter-related bacteremia in cancer patients in healthcare settings.

The clinical outcomes of patients with catheter-associated NTS bacteremia have not been well defined because of the

TA B LE 1. Clinical Manifestations of 4 Patients with Nontyphoidal Salmonella Port-Related Infections

\begin{tabular}{|c|c|c|c|c|c|c|c|c|c|}
\hline Case (year) & Age, $y$ & Sex & Underlying disease & $\begin{array}{l}\text { Healthcare- } \\
\text { Associated } \\
\text { Infection }\end{array}$ & Serogroup & Neutropenia & $\begin{array}{l}\text { Removal of } \\
\text { Catheter }\end{array}$ & Antibiotic & Mortality \\
\hline $2(2010)$ & 53 & $\mathrm{M}$ & $\begin{array}{l}\text { Esophageal cancer, } \\
\text { chronic hepatitis B }\end{array}$ & Yes & $\mathrm{D}$ & No & No & Ceftazidime & No \\
\hline $4(2011)$ & 80 & $\mathrm{~F}$ & $\begin{array}{l}\text { Rectal cancer, ovarian } \\
\text { cancer, diabetes mellitus, } \\
\text { hypertension }\end{array}$ & Yes & $\mathrm{C}$ & No & No & Ciprofloxacin & No \\
\hline
\end{tabular}


limited number of cases. In the present work, 3 patients had favorable outcomes after appropriate antibiotics treatment without removal of the port. However, 1 patient died due to NTS sepsis, even under treatment with an appropriate antibiotic. Although a firm conclusion cannot be made based on our findings, further study is warranted. Until large-scale studies investigate the clinical impact of catheter removal on the prognosis of NTS-associated, catheter-related bacteremia, clinicians may follow the recommendations by the Infectious Diseases Society of America that all devices be removed in catheter-related infections caused by Gram-negative bacteria. ${ }^{9}$

The antibiotic susceptibility patterns of the clinical isolates in this study were susceptible to all tested antibiotics, including third-generation cephalosporins and fluoroquinolones. However, in recent studies, evidence of resistance to fluoroquinolones and third-generation cephalosporins is emerging, despite the overall resistance rate of $<5 \%{ }^{10}$ Although third-generation cephalosporins or fluoroquinolones may be considered the appropriate antibiotic treatment of choice for patients with NTS bacteremia based on in vitro studies, clinicians still need to keep in mind the emergence of multidrug resistance among NST isolates.

In conclusion, port-related bacteremia caused by NTS can develop in immunocompromised patients, and NTS should be considered as a possible pathogen causing catheter-related bloodstream infections.

\section{ACKNOWLEDGMENTS}

Financial support: No financial support was provided relevant to this article. Potential conflicts of interest: All authors report no conflicts of interest relevant to this article.

\section{Hung-Jen Tang, MD; ${ }^{1,2}$ Chien-Ming Chao, MD; ${ }^{3,4}$ Chih-Cheng Lai, $\mathrm{MD}^{3}$}

Affiliation: 1. Department of Medicine, Chi Mei Medical Center, Tainan, Taiwan; 2. Department of Health and Nutrition, Chia Nan University of Pharmacy and Science, Tainan, Taiwan; 3. Department of Intensive Care Medicine,
Chi Mei Medical Center, Liouying, Tainan, Taiwan; 4. Department of Nursing, Min-Hwei College of Health Care Management, Tainan, Taiwan.

Address correspondence to Chih-Cheng Lai, Department of Intensive Care Medicine, Chi-Mei Medical Center, Liouying, Tainan, Taiwan (dtmed141@ gmail.com).

Infect Control Hosp Epidemiol 2015;36(4):492-493

(C) 2015 by The Society for Healthcare Epidemiology of America. All rights reserved. 0899-823X/2015/3604-0025. DOI: 10.1017/ice.2014.71

\section{REFERENCES}

1. Crump JA, Mintz ED. Global trends in typhoid and paratyphoid fever. Clin Infect Dis 2010;50:241-246.

2. Morpeth SC, Ramadhani HO, Crump JA. Invasive non-Typhi Salmonella disease in Africa. Clin Infect Dis 2009;49:606-611.

3. Hohmann EL. Nontyphoidal salmonellosis. Clin Infect Dis 2001;32:263-269.

4. Abuhasna S, Al Jundi A, Rahman MU, Said W. Non-typhoidal Salmonella group D bacteremia and urosepsis in a patient diagnosed with HIV Infection. J Glob Infect Dis 2012;4:218-219.

5. Ghais A, Armano R, Menten R, Mathot M, Zech F, Nassogne MC. Meningitis with subdural empyema due to non-typhoid Salmonella in a 9-month-old girl. Eur J Pediatr 2009;168:1537-1540.

6. Lai CC, Lee LN, Hsueh PR, Yu CJ, Yang PC. Empyema thoracic from Salmonella choleraesuis. Emerg Infect Dis 2005;11:1493-1494.

7. Schulze T, Lüdtke A, Rahlff I, Tunn PU, Hohenberger P. Salmonella osteomyelitis in an immunocompromised patient presenting as a primary lymphoma of the bone. Int J Infect Dis 2009;13:e67-e70.

8. Horan TC, Andrus M, Dudeck MA. CDC/NHSN surveillance definition of health care-associated infection in the acute care setting. Am J Infect Control 2008;36:309-332.

9. Mermel LA, Allon M, Bouza E, Craven E, Flynn P, O'Grady NP, et al. Clinical practice guidelines for the diagnosis and management of intravascular catheter-related infection: 2009 updated by the Infectious Diseases Society of America. Clin Infect Dis 2009;49:1-45.

10. Lunguya O, Lejon V, Phoba MF, Bertrand S, Vanhoof R, Glupczynski Y, Verhaegen J, Muyembe-Tamfum JJ, Jacobs J. Antimicrobial resistance in invasive non-typhoid Salmonella from the Democratic Republic of the Congo: emergence of decreased fluoroquinolone susceptibility and extended-spectrum beta lactamases. PLoS Negl Trop Dis 2013;7:e2103. 